\title{
Adoecimento mental dos médicos na pandemia de COVID-19
}

Mental disease among doctors in the COVID-19 pandemic

Enfermedad mental entre médicos en la pandemia COVID-19

Alexandrina Maria Augusto da Silva Meleiro - https://orcid.org/00000002-6821-2371

Arthur Hirschfeld Danila - https://orcid.org/0000-0003-1323-1994

Eduardo de Castro Humes - https://orcid.org/0000-0001-5614-8264

Sergio Pedro Baldassin - https://orcid.org/0000-0002-8954-3081

Antônio Geraldo da Silva - https://orcid.org/0000-0003-3423-7076

Edméa Fontes Oliva-Costa - https://orcid.org/0000-0002-5577-

$\underline{0454}$

\section{Resumo}

Introdução: A pandemia por COVID-19 exacerbou a angústia existencial em relação à morte. A insegurança e a incerteza relacionadas aos limites da ciência, devido à falta de consenso científico sobre essa nova doença, convocam a necessidade de investigar, refletir e revisar as informações sobre problemas de saúde mental vinculados ao trabalho médico, especialmente para os que estão na linha de frente da assistência a pessoas com COVID-19, bem como discutir o estigma relacionado ao sofrimento psíquico dessa população, em especial nessa pandemia, identificar precocemente possíveis adoecimentos psíquicos e facilitar a busca imediata de assistência em saúde mental. Métodos: Revisão narrativa visando discutir o estado da arte e atualização do conhecimento sobre o adoecimento psíquico dos médicos na pandemia de COVID-19.

Resultados: A literatura aponta que discentes, residentes, docentes e profissionais da medicina apresentam importantes prevalências de transtorno mental comum, sintomas depressivos, burnout e suicídio. A pandemia de COVID-19 apresenta um risco de aumento de prevalência de 
transtornos mentais, comumente referida como quarta onda da pandemia, associada a elementos biopsicossociais do período de quarentena $\mathrm{e}$ priorização dos cuidados físicos em detrimento dos psíquicos. Conclusões: Instituições de saúde devem realizar uma reflexão profunda sobre o seu papel na promoção, manutenção e criação de atividades e programas de prevenção do sofrimento psíquico ou transtornos mentais identificados nos profissionais médicos que nelas atuam. Estas contribuirão para o planejamento de melhores estratégias que preservem a saúde mental dos profissionais, com consequências positivas na sociedade como um todo.

Palavras-chave: Transtornos mentais, pandemias, doenças ocupacionais, esgotamento psicológico.

\section{Abstract}

Introduction: The COVID-19 pandemic exacerbated existential anguish surrounding dying. Insecurity and uncertainty related to the limits of science and the lack of scientific consensus on this new disease highlights the need to investigate, reflect, and review information on mental health problems related to medical work, especially for those in the front line of provision of care for people with COVID-19. There is also a need to discuss the stigma attached to this population's psychological suffering, especially during the pandemic and to identify mental disorders early and immediately seek specialized mental health services.

Methods: A narrative review aimed at discussing the state of the art and updating knowledge about mental illness among doctors in the COVID-19 pandemic. Results: The scientific literature reveals high prevalence rates of common mental disorder (CMD), depressive symptoms, burnout, and suicide among students, residents, teachers, and medical professionals. The COVID-19 pandemic poses a risk of mental health disorders, commonly described as the fourth wave of the pandemic, which would be triggered by biopsychosocial elements arising from the quarantine period and prioritization of physical care over provision of mental health care. Conclusions: Healthcare institutions should review and reflect on their role in the promotion, maintenance, and prevention of psychological suffering and mental disorders identified among physicians. These measures will contribute to planning of better strategies that preserve their mental health, with positive consequences for society as a whole. 
Meleiro, A.M.A.S.; Danila, A.H.; Humes, E.C.; Baldassin, S.P.; Silva, A.G.; Oliva-Costa, E.F. Keywords: Mental disorders, pandemics, occupational diseases, burnout syndrome.

\section{RESUMEN}

Introducción: La pandemia de COVID-19 exacerbó la angustia existencial que rodea a la muerte. La inseguridad y la incertidumbre relacionadas con los límites de la ciencia y la falta de consenso científico sobre esta nueva enfermedad resaltan la necesidad de investigar, reflexionar y revisar la información sobre los problemas de salud mental relacionados con el trabajo médico, especialmente para aquellos en la primera línea de la prestación de atención. para personas con COVID-19. También es necesario discutir el estigma asociado al sufrimiento psicológico de esta población, especialmente durante la pandemia, e identificar los trastornos mentales de manera temprana y buscar inmediatamente servicios de salud mental especializados. Métodos: una revisión narrativa dirigida a discutir el estado del arte y actualizar el conocimiento sobre enfermedades mentales entre los médicos en la pandemia de COVID-19. Resultados: La literatura científica revela altas tasas de prevalencia de trastorno mental común (TMC), síntomas depresivos, agotamiento y suicidio entre estudiantes, residentes, docentes y profesionales médicos. La pandemia COVID-19 presenta un riesgo de trastornos de salud mental, comúnmente descritos como la cuarta ola de la pandemia, que se desencadenaría por elementos biopsicosociales que surgen del período de cuarentena y la priorización de la atención física sobre la provisión de atención de salud mental. Conclusiones: Las instituciones de salud deben revisar y reflexionar sobre su papel en la promoción, mantenimiento y prevención del sufrimiento psicológico y los trastornos mentales identificados entre los médicos. Estas medidas contribuirán a planificar mejores estrategias que preserven su salud mental, con consecuencias positivas para la sociedad en su conjunto.

Palabras clave: Trastornos mentales, pandemias, enfermedades profesionales, síndrome de burnout.

Como citar: Meleiro, A.M.A.S.; Danila, A.H.; Humes, E.C.; Baldassin, S.P.; Silva, A.G.; Oliva-Costa, E.F. Adoecimento mental dos médicos na pandemia de COVID-19. Debates em Psiquiatria, Rio de Janeiro, 2021; 11:1-20 https://doi.org/10.25118/2763-9037.2021.v11.57 
Conflito de interesses: declaram não haver Fonte de financiamento: declaram não haver

Parecer CEP: não se aplica

Recebido em: 30/04/2021

Aprovado em: $31 / 05 / 2021$

Publicado em: 18/09/2021

\section{Introdução}

O trabalho médico se diferencia sobremaneira dos demais, na medida em que o profissional lida com a vida e a morte. O médico ainda está sujeito a agravos à sua saúde pela exposição a agentes infecciosos e químicos, a condições de trabalho insalubres e uma organização do mesmo por vezes inadequada. Está particularmente sujeito a agravos à sua saúde mental, por exercer atividade tão desencadeadora de emoções no contato diário e direto com o sofrimento humano, por possíveis identificações que advêm disso e em função das estratégias defensivas que utiliza, nem sempre de forma satisfatória ou adaptativa [1] .

A literatura apresenta um histórico importante da associação entre a prática médica e o adoecimento psíquico, desde a graduação [ㅁ- $\underline{3}$ ] e a residência [4$-\underline{5}]$, apresentando inclusive altas taxas de suicídio, maiores que as da população geral [6] .

Os elementos ambientais apresentam um papel importante entre os fatores que podem contribuir com o adoecimento psíquico, destacando-se a privação de luz solar e de sono, o ambiente com alta competitividade e as cobranças (além das próprias, as dos colegas, de pacientes e familiares). Essas populações apresentam maiores taxas de pensamento de suicídio e êxito suicida, além de baixas taxas de procura de tratamento, frequentemente recorrendo à automedicação $[\underline{3}, \underline{6}]$. 0 adoecimento aumenta o risco de o médico cometer erros e haver prejuízo do cuidado do paciente []].

A exposição a situações percebidas como graves pode gerar um conjunto de reações psicológicas, variando entre a ausência de reação ao evento, passando pela resposta proporcional, a resposta disfuncional leve e o claro adoecimento psíquico8, com a presença de diversos fatores de risco para esse adoecimento (Quadro 1). 
Meleiro, A.M.A.S.; Danila, A.H.; Humes, E.C.; Baldassin, S.P.; Silva, A.G.; Oliva-Costa, E.F.

Quadro 1 - Fatores associados a maior risco de evolução para adoecimento psiquiátrico após a exposição a trauma [ㅁ]

- Mulheres

- Infância, adolescência e terceira idade

- Pessoas com doenças clínicas e psiquiátricas prévias

- Pessoas em condições sociais prejudicadas e suporte social limitado

- $\quad$ Percepção de risco para a própria vida

- Percepção de risco para a vida de outros

- Pessoas que passam por adoecimentos físicos

- $\quad$ Situações com baixa percepção de controle e previsibilidade

- Experimentar percepção de iminência de desastre

- Experimentação de estresse desproporcional ou apresentar dissociação no momento da exposição

- $\quad$ Perda de familiares, amigos e colegas próximos

- $\quad$ Perda de propriedades e destruição de propriedade

- $\quad$ Experimentação de traumas prévios

Fonte: Adaptado de Williams et al. []]

O SARS-CoV-2, vírus causador da COVID-19, provocou mudanças em diversos contextos (pessoal, profissional, social, etc.) em todo o mundo, iniciando pela China em dezembro de 2019 e no Brasil a partir de março de 2020. A importante preocupação associada ao vírus está relacionada à sua propagação e seu potencial risco de contaminação com agravamento de sintomas, principalmente em pessoas com comorbidades e idosos, e à resposta dos sistemas de saúde [ㅁ].

O problema da sobrecarga dos sistemas de saúde por pessoas que desenvolvam COVID-19 e o risco de aumento do número de mortes diretas (pela COVID-19) e indiretas (pela incapacidade de receber o cuidado de saúde adequado por sobrecarga do sistema de saúde) estão associados ao ineditismo deste vírus junto à maior parte da população. 
Para reduzir a velocidade de propagação do vírus, foi necessária a adoção de um conjunto de mudanças de atitudes, incluindo o afastamento físico e preocupação mais intensa com a proteção individual [9 ]. Por sua vez, estas podem contribuir para um aumento dos agravos à saúde mental das pessoas, em especial dos médicos com sobrecarga e sob estresse crônico, além de preocupações acerca do risco de contaminação própria e de familiares, expostos a episódios de hostilidades contra profissionais e inúmeras mortes [10].

\section{Métodos}

Trata-se de uma revisão narrativa, apropriada para discutir o estado da arte e visando à aquisição e atualização do conhecimento sobre o adoecimento dos médicos na pandemia de COVID-19. Para a análise da produção científica, não se utilizaram técnicas qualitativas e/ou quantitativas específicas de tratamento de dados, mas a apreciação geral da qualidade, fundamentação e seriedade dos textos publicados.

\section{Resultados e Discussão}

\section{A saúde mental na pandemia}

A Associação Médica Britânica mostrou que cerca de $1 / 3$ dos médicos relatou piora de sua saúde mental com a pandemia [11] e que oferecer apoio a essa população é fundamental. Algumas especialidades já se preocuparam em investigar seus problemas durante a pandemia [12] e, de forma geral, já antecipam os problemas da segunda onda da pandemia [13].

Com a pandemia do SARS-CoV-2, causador da COVID-19, observamos um aumento da angústia existencial de todos acerca do grande tabu da humanidade que é a morte, além da insegurança e incerteza diante do novo, pelos limites da ciência, com informações desencontradas e orientações modificadas a cada nova descoberta pela falta de consenso científico [14].

Os profissionais médicos foram especialmente impactados, não apenas pelo seu papel na estruturação do sistema de saúde, muitos deles atuando nas linhas de frente, mas também pelo papel que foram conclamados a desempenhar junto à sociedade, como formadores de opinião em um momento com grandes fraturas sociais e maior radicalização dos debates.

Em tempos de COVID-19, temos convivido diariamente com o medo e a incerteza, a partir de várias informações nas mídias oriundas de órgãos 
Meleiro, A.M.A.S.; Danila, A.H.; Humes, E.C.; Baldassin, S.P.; Silva, A.G.; Oliva-Costa, E.F. oficiais de saúde no mundo e no Brasil, justamente por ser algo novo, sem resultado ainda de pesquisas científicas bem elaboradas [9] ]. Foi assim acerca do tipo de isolamento adequado, do uso de máscaras por todos e não apenas pelos profissionais da saúde e dos esquemas terapêuticos para diferentes estágios de contaminação pelo SARS-CoV-2.

Além desses estressores, observamos surgir com força o receio dos profissionais médicos em contaminar suas próprias famílias, que alimentou seus já intensos processos de cobrança pessoal e autorreprovação.

O distanciamento de familiares, como cônjuges e filhos enviados para outras localidades para permanecerem mais protegidos, gerou maior chance de se sentirem sozinhos [15].

Em alguns casos mais graves, a sensação de impotência tem sido ainda maior, como quando vistos na obrigação de facilitar o contato virtual de familiares se despedindo. Também os médicos, ao atenderem pacientes internados por SARS-CoV-2 que tiveram perdas dos pais pelo mesmo motivo, muitas vezes se sentem impotentes e se culpam, até mesmo quando enfrentam repetidas condutas burocráticas na recepção de novos pacientes.

A combinação desses novos e importantes estressores com 0 frequentemente pobre autocuidado preventivo dos profissionais médicos acarreta riscos adicionais pessoais e de saúde pública. Durante seu adoecimento, seja físico ou mental, tornam-se um risco considerável à sua própria saúde pessoal, mas também à sociedade, já que médicos desgastados emocionalmente podem se tornar menos eficientes. Há relatos de que a percepção ou o próprio destaque do "heroísmo" dos profissionais na linha de frente de combate à pandemia poderiam piorar ainda mais os riscos de excederem seus próprios limites de equilíbrio emocional e segurança [16].

Investigar e refletir sobre problemas de saúde mental vinculados ao trabalho torna-se imperativo nos dias atuais [17], especialmente para os médicos que estão na linha de frente da assistência a pessoas com suspeita ou confirmação do diagnóstico de COVID-19. Assim, é necessário revisar as evidências e discutir o estigma relacionado ao sofrimento psíquico nessa pandemia, promovendo a identificação precoce de agravos à saúde mental e a busca imediata de assistência especializada em saúde mental pelos médicos. 


\section{Mecanismos potencialmente associados ao impacto da COVID-19 na saúde mental de médicos}

Um dos primeiros textos em revista científica sobre o tema da relação da saúde mental com a COVID-19 foi o editorial do Brazilian Journal of Psychiatry, em abril de 2020 [18], onde está discutida a quarta onda potencialmente associada a essa pandemia, que se caracteriza pela eclosão de transtornos mentais. Esses transtornos acometeram a população, independentemente do contágio pelo vírus, devido a todas as consequências biopsicossociais que advêm do longo período de quarentena ou lockdown e priorização dos cuidados físicos em detrimento dos psíquicos devido ao distanciamento físico.

Para Jair Mari [19] ], o pior da pandemia se dará na área da saúde mental. Ele descreveu as várias fases da pandemia relacionadas à saúde mental e também chamou a atenção para o fato de que, embora todos estejam submetidos a estressores imprescindíveis, nem todos irão desenvolver transtornos mentais, uma vez que outras variáveis são necessárias para o desenvolvimento de um adoecimento, como fatores biológicos, a capacidade de resiliência individual, o suporte de apoio familiar e institucional, a identificação precoce de sinais e sintomas e intervenção preventiva adequada [19].

Para o autor, haveria impacto em saúde mental durante quatro fases, com mecanismos diversos para a ação. A primeira fase é marcada pelo foco no medo de ser contaminado ou de contaminar outras pessoas, sendo esta uma reação natural, mas que não é diferente de outras situações agudas traumáticas causadas por outras patologias infecciosas onde este risco de contaminação também existe; entretanto, o desconhecimento sobre a COVID-19 exacerba este medo.

Na segunda fase, relacionada com o afastamento físico compulsório, ocorre uma mudança coerciva de rotina. Daí observamos uma reação de ajustamento situacional diante das circunstâncias de insegurança e do medo que nos prepara o futuro, caracterizada por tristeza, ansiedade, angústia, irritabilidade e desconforto em relação à nova realidade, mas ainda sem comprometimento das atividades diárias, seja no trabalho remoto, presencial ou no cuidado pessoal e dos familiares para a preservação da saúde integral.

Contudo, é indispensável estar atento aos sintomas para diferenciar uma reação normal de uma reação patológica, seja pela importante intensidade 
Meleiro, A.M.A.S.; Danila, A.H.; Humes, E.C.; Baldassin, S.P.; Silva, A.G.; Oliva-Costa, E.F. de sintomas, seja pelo progressivo aumento que pode ocorrer na reação patológica. Essa diferenciação determina a necessidade de avaliação por um profissional especializado, que poderá recomendar modificações possíveis e saudáveis de rotina, que priorizem as estratégias preventivas contra agravos à saúde física e psíquica igualmente.

$\mathrm{Na}$ terceira fase proposta, o ponto central são as mortes abruptas decorrentes da COVID-19, sem os rituais habituais de despedida que favorecem a elaboração das perdas e vivência do luto normal. O autor ainda discute o impacto a que os profissionais de saúde da linha de frente são submetidos. Destaca o alto nível de exigência física e emocional, dentro de uma estrutura assistencial insuficiente para garantir segurança profissional.

São vários os relatos da carência de equipamentos de proteção individual, levando, além do medo de ser contaminado, a uma sensação de descaso, desamparo e frustração com as condições adversas de trabalho, sem contar a convivência com cenas terrificantes no dia a dia. Nessa situação, espera-se um nível elevado da síndrome de burnout, associada ao esgotamento profissional [19].

Em um comentário publicado online, em 21 de abril de 2020, no periódico Lancet Psychiatry, os membros da International COVID-19 Suicide Prevention Research Collaboration alertaram sobre o possível aumento das taxas de suicídio à medida que a pandemia se espalha, e os seus efeitos de longo prazo na economia, na população em geral e nos grupos mais vulneráveis se tornam mais claros [20].

Essa preocupação ecoa em relatos como o de uma médica coordenadora de UTI em Manhattan (NY), que não tinha antecedentes de transtornos mentais, segundo o seu pai também médico, e que cometeu suicídio em 26 de abril de 2020 em Charlottesville, nos EUA, quando retornou ao trabalho após se recuperar da contaminação por COVID-19 e a necessária quarentena. Segundo a família, a médica chegou a trabalhar 18 horas por dia e a dormir nos corredores do hospital [21]; por isso a importância de cuidar do cuidador.

Muitas das características psicodinâmicas que conduzem as pessoas para a carreira médica também as predispõem para os distúrbios emocionais descritos na literatura. Além de comprometer a relação médico/paciente e o comportamento ético do médico, elas podem desencadear vários 
processos de adoecimento e até justificar os elevados índices de suicídio encontrados nesse grupo [르].

Uma das motivações para a escolha da carreira médica é o desejo de vencer a morte, o que faz o estudante assumir compromissos onipotentes durante a sua formação. O confronto com os limites da realidade da profissão gera sentimento de culpa e impotência, que favorecem o surgimento de quadros depressivos ou ansiosos.

É necessário, ao médico já em exercício da profissão, um programa de conscientização e orientação de que a informação técnica anteriormente adquirida não the dá imunidade aos conflitos emocionais, assim como um bom preparo de profissionais para lidar com médicos em risco de suicídio, pois os sentimentos positivos e negativos da contratransferência se misturam, principalmente os de identificação [22].

A literatura já documentou previamente a associação entre transtornos mentais e pioras dos indicadores de qualidade de cuidado, absenteísmo, presenteísmo e abandono de vínculos empregatícios [22].

Tendo em vista o potencial impacto sobre os sistemas de saúde do mundo, que muitas vezes já funcionam com dimensionamento mínimo de profissionais, o cuidado com a saúde mental dos profissionais é essencial. Para isso é central que ocorra não apenas o apoio dos gestores das instituições de saúde, mas também apoio mútuo no cuidado solidário.

A observação e atenção para sinais e sintomas que indiquem agravos à saúde física e/ou psíquica de qualquer membro da equipe terapêutica é fundamental. Devemos ter em conta que qualquer perda por morte ou afastamento por doença provoca desestruturação nos serviços, mas também nas famílias dos profissionais, no cuidado da comunidade e na equipe [22].

\section{Estado atual de evidências}

Essas associações apresentam consonância com os achados da literatura, que mostram que os profissionais envolvidos apresentam altas taxas de sintomatologia depressiva e ansiosa [23-24], sendo que os que estão na linha de frente [25], e em regiões com maior incidência de quadros de COVID-19, apresentam maior risco de apresentar sintomatologia importante.

Um estudo para avaliar a prevalência e os preditores de depressão, ansiedade e sua comorbidade entre trabalhadores essenciais do Brasil e da 
Meleiro, A.M.A.S.; Danila, A.H.; Humes, E.C.; Baldassin, S.P.; Silva, A.G.; Oliva-Costa, E.F. Espanha, como profissionais de saúde durante a pandemia COVID-19, mostrou que morar no Brasil foi associado com aumento de maior chance de depressão, ansiedade e ambos quando comparado a morar na Espanha [24].

Esses trabalhadores, apesar de proporções importantes de sintomatologia depressiva, apresentam pouca procura por serviços de atenção à saúde mental, apesar de estarem expostos a uma importante quantidade de materiais e serviços de divulgação de temas em saúde mental, mantendo a dissociação entre o adoecimento e a busca efetiva de tratamento.

A presença de patologia psiquiátrica conhecida prévia está associada a maior risco de exacerbação da sintomatologia durante a pandemia entre médicos [26]. As evidências, entretanto, ainda são limitadas frente ao curto tempo entre o início da pandemia e o momento atual.

Individualmente, entre os principais eixos de atividades que podemos tomar e recomendar, temos os itens elencados no Quadro 2, com destaque na promoção de saúde, seja física ou mental [27]. Entretanto, ações para cuidado e detecção precoce do adoecimento de médicos no ambiente de trabalho são essenciais (Quadro 3), em especial para permitir o diagnóstico precoce e o adequado seguimento, evitando o abandono de tratamento e o autotratamento.

Quadro 2 - Principais estratégias preventivas individuais baseadas nas orientações atuais do Ministério da Saúde e FIOCRUZ [르]

- $\quad$ Acolher e reconhecer seus medos como sendo naturais, bem como o choro em situações específicas, procurando pessoas de confiança para conversar;

- Resgatar instrumentos de cuidado para controle emocional que já deram certo no passado em situações críticas;

- $\quad$ Realizar atividades que ajudem na redução do nível de estresse agudo e com as quais se identifique;

- Garantir pausas sistemáticas durante o trabalho e entre os turnos, se possível em espaço relaxante;

- $\quad$ Caso seja estigmatizado por medo de contágio, compreenda que não é pessoal, mas fruto do medo e do estresse causado pela pandemia, e dessa forma procure ajuda de colegas de trabalho e supervisores que

11 Debates em Psiquiatria, Rio de Janeiro, 2021, 11:1-20 https://doi.org/10.25118/2763-9037.2021.v11.57 
possam compartilhar das mesmas dificuldades, buscando soluções compartilhadas;

- Investir em ações compartilhadas de cuidado com seus pares;

- Continuar elaborando bons projetos de vida pessoal e profissional, adaptando-os às condições associadas à pandemia;

- Manter ativa a rede socioafetiva, estabelecendo contato frequente, mesmo que virtual, com familiares, amigos e colegas, entendendo que o distanciamento físico não implica em isolamento social;

- Evitar o uso do tabaco, álcool ou outras drogas, em especial como ferramenta para lidar com as emoções;

- Buscar um profissional de saúde mental (psicólogo e/ou psiquiatra) quando as estratégias utilizadas não estiverem sendo suficientes para a sua estabilização emocional;

- Buscar fontes confiáveis de informação, como o site da Organização Mundial da Saúde;

- Reduzir o tempo que passa assistindo noticiário fixado na pandemia;

- Compartilhar as ações e estratégias de cuidado e solidariedade, a fim de aumentar a sensação de pertença e conforto social;

- Estimular o espírito solidário e incentivar a participação da comunidade.

FIOCRUZ = Fundação Oswaldo Cruz.

Fonte: Adaptado de Ministério da Saúde/FIOCRUZ [27].

Quadro 3 - Principais síndromes, sintomas e diagnósticos relacionados à pandemia entre profissionais de saúde [27]

Contexto associado

Ansiedade Torna-se mais intensa com o passar do tempo, e traumáticos

\section{Diagnósticos}

mais

associados

Insônia; pesadelos

Transtorno de

ansiedade

generalizada

(TAG), 
Meleiro, A.M.A.S.; Danila, A.H.; Humes, E.C.; Baldassin, S.P.; Silva, A.G.; Oliva-Costa, E.F.

\begin{tabular}{|c|c|c|}
\hline $\begin{array}{l}\text { o foco do } \\
\text { estresse vai } \\
\text { além da } \\
\text { pandemia e } \\
\text { compromete } \\
\text { outros } \\
\text { aspectos da } \\
\text { vida familiar, } \\
\text { conjugal e } \\
\text { profissional }\end{array}$ & $\begin{array}{l}\text { recentes; sensação } \\
\text { de taquicardia; } \\
\text { somatização de } \\
\text { sintomas físicos, } \\
\text { principalmente } \\
\text { falta de ar, } \\
\text { associados à } \\
\text { contaminação por } \\
\text { COVID-19; excesso } \\
\text { de higienização } \\
\text { que limite a } \\
\text { realização de } \\
\text { outras atividades } \\
\text { necessárias ou } \\
\text { provoque } \\
\text { escoriação na pele }\end{array}$ & $\begin{array}{l}\text { transtorno de } \\
\text { estresse pós- } \\
\text { traumático } \\
\text { (TEPT), } \\
\text { transtorno } \\
\text { obsessivo- } \\
\text { compulsivo } \\
\text { (TOC), } \\
\text { transtorno } \\
\text { dissociativo }\end{array}$ \\
\hline
\end{tabular}

Depressão

Aumento da Depressão

tristeza; deixar de

ter interesse pelas

atividades de que

gostava;

irritabilidade

descontrolada;

sensação de

fadiga; desgaste

emocional; insônia;

pensamentos

negativos; ideias

de que não vale a

pena viver

Fonte: Adaptado de Ministério da Saúde/FIOCRUZ [27].

Uma medida potencial, simples e efetiva é a criação de núcleos de atendimento e de apoio médico psiquiátrico e psicológico aos profissionais da saúde. Já há experiência relatada na literatura, com mais de 10 anos de suporte através de ambulatório didático para internos no contexto de um hospital-escola. A partir dessa vivência, além do cuidado dos 
profissionais/pacientes, os internos aprendem e podem refletir sobre as histórias que conhecem.

A intervenção terapêutica deve ser imediata, pelo risco de suicídio, como já ocorreram casos de profissionais da saúde nessa pandemia em várias partes do mundo. Daí a importância do diagnóstico precoce, quando os sintomas depressivos ainda são leves, para que o tratamento possa evitar a evolução do quadro para a depressão grave ou o suicídio, o que seria uma perda irreparável e extremamente traumática para toda a comunidade [28].

Há pouca literatura sobre ações sistêmicas tomadas para a prevenção, diagnóstico precoce e prevenção do afastamento desses profissionais, tendo destaque iniciativas nacionais do Ministério da Saúde [29] e do Hospital das Clínicas da Faculdade de Medicina da Universidade de São Paulo (HCFMUSP) [30], além de iniciativas de grupos de profissionais, como alguns colegas vinculados à Associação Mineira de Psiquiatria, uma afiliada da Associação Brasileira de Psiquiatria (ABP), e a parceria entre a ABP e o Ministério da Saúde. O Ministério da Saúde firmou parceria com a ABP para garantir atendimento psiquiátrico aos profissionais do Sistema Único de Saúde (SUS) que estão na linha de frente do combate à COVID19.

O Amazonas foi um dos primeiros estados atendidos, e o presidente da ABP informou que cerca de 900 mil profissionais do SUS vão receber questionários "para saber sobre a saúde mental deles, com a preocupação do tipo cuidando do cuidador". A ABP apoia também outra sondagem sobre a saúde mental do povo brasileiro, para identificar se haverá modificação do padrão de incidência de transtornos mentais nesse período de pandemia [18].

\section{Conclusões}

Acreditamos que a reflexão profunda das instituições de saúde sobre o seu papel na promoção, manutenção e a criação de atividades e de programas de prevenção do sofrimento psíquico ou transtornos mentais identificados nos profissionais médicos contribuirá para o planejamento de melhores estratégias que preservem a saúde mental dos mesmos, com consequências positivas na saúde dessa população, de seus familiares e pacientes e, ainda, em saúde pública. Afinal, é indispensável reforçar que uma boa saúde mental dos médicos vai depender principalmente dos 
Meleiro, A.M.A.S.; Danila, A.H.; Humes, E.C.; Baldassin, S.P.; Silva, A.G.; Oliva-Costa, E.F. gestores de instituições de saúde que precisam priorizar e valorizar o "cuidado a quem cuida".

\section{Referências}

1. Baldassin S. O desgaste no internato: o nascimento do coping de um médico. In: Guimarães KB. Saúde mental do médico e do estudante de medicina. São Paulo: Casa do Psicólogo; 2007. p. 51-60. https://www.pearsonclinical.com.br/saude-mental-do-medico-e-doestudante-de-medicina.html

2. Pacheco JP, Giacomin HT, Tam WW, Ribeiro TB, Arab C, Bezerra IM, et al. Mental health problems among medical students in Brazil: a systematic review and meta-analysis. Braz J Psychiatry. 2017;39:369-78. https://doi.org/10.1590/1516-4446-2017-2223 PMid:28876408 MCid:PMC7111407

3. Rotenstein LS, Ramos MA, Torre M, Segal JB, Peluso MJ, Guille C, et al. Prevalence of depression, depressive symptoms, and suicidal ideation among medical students: a systematic review and metaanalysis. JAMA. 2016;316:2214-36.

https://doi.org/10.1001/jama.2016.17324 PMid:27923088 PMCid:PMC5613659

- 4. Mata DA, Ramos MA, Bansal N, Khan R, Guille C, Di Angelantonio $\mathrm{E}$, et al. Prevalence of depression and depressive symptoms among resident physicians: a systematic review and meta-analysis. JAMA. 2015;314:2373-83. https://doi.org/10.1001/jama.2015.15845 PMid:26647259 PMCid:PMC4866499

5. Rodrigues TM, Melo EV, Oliva-Costa EF. Prevalência da síndrome de Burnout e fatores associados entre médicos egressos de universidade pública brasileira. In: 170 Congresso da ISMA-BR. 2017. Anais do ISMA-BR. http://www.ismabrasil.com.br/congressos/congresso2017/patrocine

6. Duarte D, El-Hagrassy MM, Couto TC, Gurgel W, Fregni F, Correa $\mathrm{H}$. Male and female physician suicidality: a systematic review and meta-analysis. JAMA Psychiatry. 2020;77:587-97. 
https://doi.org/10.1001/jamapsychiatry.2020.0011 PMid:32129813 PMCid:PMC7057173

- 7. Pereira-Lima K, Mata DA, Loureiro SR, Crippa JA, Bolsoni LM, Sen $\mathrm{S}$. Association between physician depressive symptoms and medical errors: a systematic review and meta-analysis. JAMA Netw Open. 2019;2:e1916097.

https://doi.org/10.1001/jamanetworkopen.2019.16097 PMid:31774520 PMCid:PMC6902829

8. Williams R, Bisson J, Kemp V. Principles for responding to people's psychosocial and mental health needs after disasters [Internet]. The Royal College of Psychiatrists. 2014 Nov [cited 2020 Mar 31]. https://www. apothecaries.org/wpcontent/uploads/2019/02/OP94.pdf

9. World Health Organization (WHO). Infection prevention and control during health care when novel coronavirus ( $\mathrm{nCoV}$ ) infection is suspected [Internet]. 2020 Mar 19 [cited 2021 Jun 10]. https://www.who.int/publications/i/item/10665-331495

10. Zhang C, Yang L, Liu S, Ma S, Wang Y, Cai Z, et al. Survey of insomnia and related social psychological factors among medical staff involved with the 2019 novel coronavirus disease outbreak. Front Psychiatry. 2020;11:306.

https://doi.org/10.3389/fpsyt.2020.00306 PMid:32346373

PMCid:PMC7171048

- 11. Torjesen I. Covid-19: doctors need proper mental health support, says BMA. BMJ. 2020;369:m2192.

https://doi.org/10.1136/bmj.m2192 PMid:32482682

- 12. Shah N, Raheem A, Sideris M, Velauthar L, Saeed F. Mental health amongst obstetrics and gynaecology doctors during the COVID-19 pandemic: results of a UK-wide study. Eur J Obstet Gynecol Reprod Biol. 2020;253:90-4.

https://doi.org/10.1016/j.ejogrb.2020.07.060 PMid:32829231 PMCid:PMC7417944

- 13. Rimmer A. Covid-19: two fifths of doctors say pandemic has worsened their mental health. BMJ. 2020;371:m4148.

https://doi.org/10.1136/bmj.m4148 PMid:33109530 
Meleiro, A.M.A.S.; Danila, A.H.; Humes, E.C.; Baldassin, S.P.; Silva, A.G.; Oliva-Costa, E.F.

14. Lazzerini M, Putoto G. COVID-19 in Italy: momentous decisions and many uncertainties. Lancet Glob Health. 2020;8:e641-2. https://doi.org/10.1016/S2214-109X(20)30110-8

15. Rathod S, Pallikadavath S, YoungAH, Graves L, Rahman MM, Brooks A, et al. Psychological impact of COVID-19 pandemic: protocol and results of first three weeks from an international crosssection survey - focus on health professionals. J Affect Disord Rep. 2020;1:100005. https://doi.org/10.1016/j.jadr.2020.100005 PMid:33313579 PMCid:PMC7507987

16. Rimmer A. Covid-19: drop the hero narrative and support doctors' mental health, says charity. BMJ. 2021;372:n337. https://doi.org/10.1136/bmj.n337 PMid:33541858

17. Supporting the mental health of NHS staff. The role of NHS leaders in reducing mental health stigma and creating mentally healthy cultures [Internet]. 2021 [cited 2021 Jun 10].

https://cdn.mentalhealthatwork.org.uk/wpcontent/uploads/2021/02/02114357/BMA-Stigma-Resource-.pdf

18. da Silva AG, Miranda DM, Diaz AP, Telles AL, Malloy-Diniz LF, Palha AP. Mental health: why it still matters in the midst of a pandemic. Braz J Psychiatry. 2020;42:229-31.

https://doi.org/10.1590/1516-4446-2020-0009 PMid:32267344 PMCid:PMC7236155

19. Mari J. O pior da pandemia se dará na saúde mental [Internet]. Folha de São Paulo. 2020 [cited 2020 Jun 08]. https://www1.folha.uol.com.br/opiniao/2020/05/o-pior-dapandemia-se-dara-na-saude-mental.shtml

20. Gunnell D, Appleby L, Arensman E, Hawton K, John A, Kapur N, et al. Suicide risk and prevention during the COVID-19 pandemic [Internet]. 2020 [cited 2020 Sep 28].

https://www.thelancet.com/journals/lanpsy/article/PIIS2215-

0366(20)30171-1/fulltext

21. Coluna Mundo. Médica da linha de frente contra coronavírus comete suicídio nos EUA [Internet]. Folha de São Paulo. 2020 [cited 2020 Jun 13]. 
https://www1.folha.uol.com.br/mundo/2020/04/medica-da-linhade-frente-contra-coronavirus-comete-suicidio-nos-eua.shtml

- 22. Meleiro AM. Consequências do trabalho na saúde mental do médico: qual a realidade? In: Cordeiro Q, Razzouk D, Lima MG. Trabalho e saúde mental dos profissionais da saúde. São Paulo: CRMESP; 2016. p. 107-30. https://sbph.org.br/wpcontent/uploads/2014/03/trabalho-e-saude-mental-dosprofissionais-da-saude.pdf

23. Pappa S, Ntella V, Giannakas T, Giannakoulis VG, Papoutsi E, Katsaounou P. Prevalence of depression, anxiety, and insomnia among healthcare workers during the COVID-19 pandemic: a systematic review and meta-analysis. Brain Behav Immun. 2020;88:901-7. https://doi.org/10.1016/j.bbi.2020.05.026 PMid:32437915 PMCid:PMC7206431

24. De Boni RB, Balanzá-Martínez V, Mota JC, Cardoso TA, Ballester P, Atienza-Carbonell, B, et al. Depression, anxiety, and lifestyle among essential workers: a web survey from Brazil and Spain during the COVID-19 pandemic. J Med Internet Res. 2020;22:e22835. https://doi.org/10.2196/22835 PMid:33038075 PMCid:PMC7641648

25. Badahdah A, Khamis F, Al Mahyijari N, Al Balushi M, Al Hatmi H, Al Salmi I, et al. The mental health of health care workers in Oman during the COVID-19 pandemic. Int J Soc Psychiatry. 2021;67:90-5. https://doi.org/10.1177/0020764020939596 PMid:32635837 PMCid:PMC8191150

- 26. Civantos AM, Bertelli A, Gonçalves A, Getzen E, Chang C, Long $Q$, et al. Mental health among head and neck surgeons in Brazil during the COVID-19 pandemic: a national study. Am J Otolaryngol. 2020;41:102694. https://doi.org/10.1016/j.amjoto.2020.102694 PMid:32854041 PMCid:PMC7442010

27. Ministério da Saúde, FIOCRUZ. Saúde mental e atenção psicossocial na pandemia Covid-19 [Internet]. [cited 2020 Jun 15]. https://www.fiocruzbrasilia.fiocruz.br/wpcontent/uploads/2020/04/Sa\%C3\%BAde-Mental-e- 
Meleiro, A.M.A.S.; Danila, A.H.; Humes, E.C.; Baldassin, S.P.; Silva, A.G.; Oliva-Costa, E.F. Aten $\%$ C3\%A7\%C3\%A3o-Psicossocial-na-Pandemia-Covid-19recomenda\%C3\%A7\%C3\%B5es-gerais.pdf

- 28. Galbraith N, Boyda D, McFeeters D, Hassan T. The mental health of doctors during the COVID-19 pandemic. BJPsych Bull. 2021;45:93-7. https://doi.org/10.1192/bjb.2020.44 PMid:32340645 PMCid:PMC7322151

29. Salum GA, Rehmenklau JF, Csordas MC, Pereira FP, Castan JU, Ferreira $A B$, et al. Supporting people with severe mental health conditions during the COVID-19 pandemic: considerations for low- and middleincome countries using telehealth case management. Braz J Psychiatry. 2020;42:451-2. https://doi.org/10.1590/1516-4446-2020-1078 PMid:32578690 PMCid:PMC7430400

- 30. Fukuti P, Uchôa CL, Mazzoco MF, Corchs F, Kamitsuji CS, De Rossi $L$, et al. How institutions can protect the mental health and psychosocial well-being of their healthcare workers in the current COVID19 pandemic. Clinics (Sao Paulo). 2020;75:e1963.

https://doi.org/10.6061/clinics/2020/e1663 


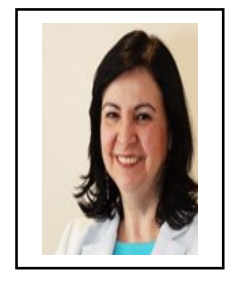

Alexandrina Maria Augusto da Silva Meleiro $\underline{\text { ORCID } \text { Lattes }}$

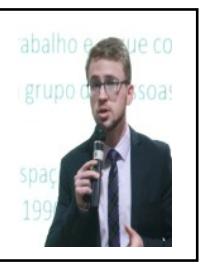

Arthur Hirschfeld Danila

ORCID Lattes

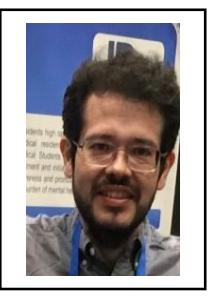

Eduardo de Castro Humes

ORCID Lattes

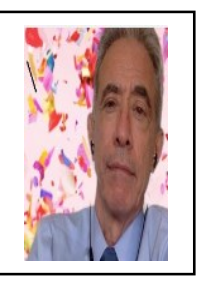

Sergio Pedro Baldassin

ORCID Lattes

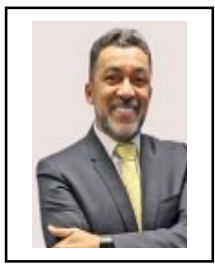

Antônio Geraldo da Silva $\underline{\text { ORCID Lattes }}$

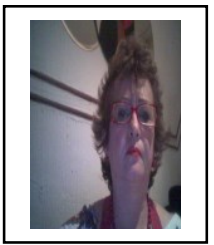

Edméa Fontes Oliva-Costa

ORCID Lattes 